\title{
Usando Smartphones, QR Code e Game of Thrones para Gamificar o Ensino e Aprendizagem de Termometria
}

\author{
Denis A. Silva ${ }^{1}$, Raquel R. Dias ${ }^{2}$, Vania de Fátima T. Flippert ${ }^{3}$, Clodis Boscarioli ${ }^{1}$ \\ ${ }^{1}$ Pós-Graduação Stricto Sensu em Ensino (PPGEn) - Nível de Mestrado - Universidade \\ Estadual do Oeste do Paraná (UNIOESTE) - Av. Tarquino J. dos Santos, 1300 - Jardim \\ Universitário - Foz do Iguaçu - PR - Brasil \\ ${ }^{2}$ Colégio Estadual Monsenhor Guilherme \\ Rua Naipi, 261 - Centro - Foz do Iguaçu - Paraná - Brasil \\ ${ }^{3}$ Colégio Estadual Monteiro Lobato \\ Rua Vereador Rubino Pasquetti, 555 - Centro - Céu Azul - Paraná - Brasil \\ \{denis_okizy@yahoo.com.br, rdrodrigues23@gmail.com, \\ vaniaf́lippert@bol.com.br, boscarioli@gmail.com\}
}

\begin{abstract}
This paper aims to present the experience of the application of a pedagogical activity (dynamic game) designed to fix the contents of Physics, especially those of Thermometry worked in the classroom with second year students of a public school using QR Code technology, gamification elements, the incentive to mobile learning using the students' smartphones and their interests through the Game of Thrones series. It was found that activity such as this motivates and fosters cooperation among students, helps to overcome the lack of technological structure in the school and enables new insights about teaching-learning through mobile devices.
\end{abstract}

Resumo. Este artigo objetiva apresentar a experiência da aplicação de uma atividade pedagógica (dinâmica de jogo) elaborada com o intuito de fixar os conteúdos da Física, especialmente os de termometria, trabalhados em sala de aula com alunos do segundo ano do Ensino Médio de uma escola pública, por intermédio do uso da tecnologia QR Code, de elementos da gamificação, do incentivo à aprendizagem móvel usando os celulares dos alunos e de seus interesses pela série Game of Thrones. Constatou-se que atividade como essa motiva e fomenta a cooperação entre os estudantes, auxilia na superação da falta de estrutura tecnológica na escola e possibilita novos olhares sobre o ensino-aprendizagem por meio de dispositivos móveis.

\section{Introdução}

Desde o último quarto do século XX, vive-se em um acelerado processo de quebras de paradigmas, principalmente, no que tange ao desenvolvimento tecnológico. Na proporção que as Tecnologias da Informação e da Comunicação (TDIC) vão se inserindo na sociedade, provocam-na a mudar "suas formas de organizar-se, de produzir bens, de comercializá-los, de divertir-se, de ensinar e de aprender" [Moran, 2006].

Com a efervescência tecnológica e as políticas de democratização do uso e do acesso às TDIC, o homo digitalis tem a possibilidade estabelecer novas formas de se 
relacionar com o mundo que o cerca. A educação, por conseguinte, não está alheia a esse processo de mudanças. "O uso de tecnologias e dispositivos digitais para ampliar o acesso à educação de qualidade é um fenômeno em franca expansão e convida a uma profunda reflexão sobre o futuro dos processos de ensino e aprendizagem" [Brandão e Vargas, 2016, p. 9].

No século XXI, a inserção de TDIC no processo de ensino e aprendizagem já é uma consoante não passível de rechaço. Pressiona-se, cada vez mais, a incorporação de metodologias que as integrem a métodos de ensino ativos que sejam capazes de gerar aprendizagem significativa para a geração de alunos digitais que estão nas instituições de ensino atualmente.

Dentre os desafios que essa integração traz - especialmente à rede pública de ensino, está a desigualdade socioeconômica entre os estudantes e a infraestrutura tecnológica inadequada ou por muitas vezes, ausente. Conforme Moraes e Varela (2007), a falta de atividades diversificadas, professores não capacitados, as condições físicas das escolas são alguns problemas entre muitos que levam os alunos à desmotivação.

A aprendizagem móvel - M-Learning - por meio da utilização de equipamentos tecnológicos que os próprios estudantes possuem é uma das opções a serem exploradas pelos educadores como alternativa à falta de infraestrutura tecnológica e como meio à diversificação das atividades. Essa ideia é conhecida como Bring Your Own Device (BYOD) - "Traga seu próprio dispositivo" em português:

BYOD (bring your own device), também chamado de BYOT (bring your own technology), é o nome que se dá a política que permite que funcionários levem seus próprios dispositivos móveis (notebooks, tablets, smartphones) para o espaço de trabalho para usá-los com os próprios aplicativos e informações da empresa. $\mathrm{Na}$ escola funciona da mesma maneira. Os gestores pedem que os alunos e professores tragam seus próprios dispositivos para que eles possam ser usados em sala de aula. [Porvir, 2015]

Franco et al. (2016) destaca que as multifuncionalidades dos dispositivos móveis como os dicionários, câmeras, jogos, calculadoras, Bluetooth, SMS podem favorecer tanto a comunicação e a aprendizagem no âmbito escolar quanto fora dele. Ademais, são tecnologias sem custo para as escolas.

Bastos, Rapkiewicz \& Benvenuti (2016) e Franco et al. (2016) ressaltam que os dispositivos móveis, em especial os celulares, costumam ser proibidos em sala de aula e são considerados como objetos de distração dos alunos. Entretanto, essas autoras argumentam que a incorporação desses dispositivos no processo de aprendizagem pode dar novos significados às tecnologias, diminuir a distração e ao mesmo tempo, motivar os estudantes.

No que tange à desmotivação, a gamificação tem sido utilizada no ambiente escolar para elevar a motivação em sala de aula. A sua finalidade é "potencializar a motivação, a concentração, o esforço, a fidelização e outros valores positivos comuns a todos os jogos. Trata-se de uma nova e poderosa estratégia para influenciar e motivar grupos de pessoas" [Gamificación, 2013]. A gamificação usa elementos de jogos e técnicas de projeto de jogo em contextos não convencionais de jogos [Ferreira et al., 2016, 
p. 151]. Há aqui, o desafio para o professor de transformar esses equipamentos e estratégias em recursos pedagógicos a serviço da aprendizagem e da comunicação.

O objetivo desse trabalho é descrever a criação e a aplicação de uma atividade pedagógica elaborada para fixar os conteúdos da Física, especialmente os de termometria, trabalhados em sala de aula de forma ativa, motivadora e significativa por intermédio do uso da tecnologia $Q R$ Code, de elementos da gamificação, do incentivo à aprendizagem móvel e do interesse dos alunos pela série Game of Thrones ${ }^{1}$. A partir disso, desenvolveuse uma dinâmica de jogo (processo de ensino gamificado) no qual os estudantes do segundo ano do Ensino Médio foram estimulados a utilizarem os seus smartphones como um instrumento pedagógico para fazer a leitura e caça de $Q R$ Codes espalhados pela escola que representaram as "Casas dos Setes Reinos de Westeros" da série Game of Thrones e que continham temperaturas a serem transformadas para determinadas escalas termométricas. O prêmio foi a conquista do "Trono de Ferro"- símbolo do poder e do controle dos Sete Reinos de Westeros - condicionada ao maior número correto de conversões das temperaturas capturadas para suas respectivas escalas termométricas.

Este documento segue assim organizado: A Seção 2 aborda elementos teóricos, conceituais sobre Termometria e $Q R$ Code, provendo ao leitor subsídios necessários à compreensão do trabalho ora proposto. $\mathrm{Na}$ Seção 3 os materiais e métodos são descritos. A Seção 4 traz a análise e discussão dos resultados da pesquisa e, por fim, na Seção 5 constam as conclusões e perspectivas da pesquisa.

\section{Referencial Teórico}

Apresentar-se-á uma breve fundamentação teórica acerca da termometria e da tecnologia QR Code nesta seção.

\subsection{Termometria}

Desde o início do século XIX, a temperatura é reconhecida como um dos pontos chaves do ensino básico de Física dada sua importância na caracterização e determinação de inúmeros fenômenos físicos, químicos e biológicos [Pires, 2006, p. 105].

A temperatura é uma das grandezas físicas mais conhecidas e citadas que está inserida no cotidiano das pessoas por meio dos jornais, rádios, televisão, previsões meteorológicas, equipamentos eletrodomésticos etc. Pode-se percebê-la de várias maneiras, por exemplo, para saber quão quente ou frio está um determinado corpo em relação a outro corpo de referência, ou ainda como indicadora do sentido da troca de energia na forma de calor entre o corpo e sua vizinhança [Marques, 2009].

Para isso, as escalas foram criadas por cientistas e fabricantes de termômetros com o objetivo de padronizá-las para que facilmente fossem reproduzidas e convertidas em qualquer país e lugar. Algumas propostas se mostraram consistentes e foram adotadas e universalizadas chegando aos tempos atuais nas graduações dos termômetros, na literatura e nas linguagens técnica e coloquial [Analógica, 2013].

No Ensino Médio, as escalas Celsius, Kelvin e Fahrenheit são as mais trabalhadas a fim de que os estudantes as identifiquem, compreendam as relações entres elas e

\footnotetext{
${ }^{1}$ Série de TV estadunidense baseada na série de livros de Crônicas de Gelo e Fogo de George R. R. Martin. Na série, o "Trono de Ferro" que representa o domínio dos Sete Reinos de Westeros é disputado pelas famílias em jogo de alianças, guerras, conspirações etc.
} 
percebam suas aplicações no cotidiano por intermédio de suas equações.

As Equações 1, 2 e 3 são usadas para transformar uma temperatura da escala Celsius para Fahrenheit ou vice-versa, para transformações entre as escalas Celsius e Kelvin e para transformar temperaturas entre as escalas Kelvin e Fahrenheit, respectivamente.

$$
\begin{aligned}
& \mathrm{TC} / 5=\mathrm{TF}-32 / 9 \\
& \mathrm{TK}=\mathrm{TC}+273 \\
& \mathrm{TK}-273 / 5=\mathrm{TF}-32 / 9
\end{aligned}
$$

A sigla TC corresponde à temperatura em Celsius, assim como TF e TK correspondem às temperaturas em Fahrenheit e Kelvin, respectivamente.

Essas também foram as equações escolhidas para a conversão das temperaturas e suas respectivas escalas coletadas por meio dos $Q R$ Codes na dinâmica com os alunos.

\subsection{Tecnologia $Q R$ Code}

O QR Code (Quick Response Code) é uma espécie de código de barras bidimensional, criado em 1994 pela empresa japonesa Denso Wave. Mesmo em baixas resoluções, como imagens feitas por câmeras VGA encontradas nos celulares mais básicos, o código pode ser interpretado rapidamente e sem erros [LIMA et al., 2015].

Com as inúmeras possibilidades de aplicações dessa tecnologia, constata-se que o seu uso na Educação poderá ser de grande importância, pois revolucionará a forma como estudantes poderão entrar em contato com informações de forma rápida e prática, e principalmente em lugares voltados para a educação [Delinski et al., 2012].

Na prática, o QR Code significou a evolução do código de barras, permitindo armazenar diferentes tipos de dados, como links, imagens, formulários, Short Message Service (SMS), textos, entre outras informações [Araújo, 2015].

O uso das Tecnologias da Informação Móveis e Sem Fio (TIMS) aumenta os desafios da realidade escolar. Educadores precisam cada vez mais se adequarem à realidade desenhada pelas TIMS, dentre as quais o smartphone, um aparelho popular que pode conter aplicativos otimamente utilizados em sala de aula como recurso pedagógico [Araújo, 2015].

À luz dessas tecnologias, buscou-se na atividade relatada desenvolver $Q R$ Codes para o espaço físico do colégio, armazenando informações sobre o conteúdo de Termometria com objetivo de viabilizar a aplicação desta tecnologia na disseminação e propagação do conhecimento, criando um ambiente físico e virtual dentro e fora da sala de aula. Essa é uma contribuição desse trabalho, ou seja, contextualizar e demonstrar na prática como os professores poderão tornar essa ferramenta em um recurso pedagógico, ou ainda, ser mais uma evidencia de como se pode potencializar o dispositivo móvel no processo de ensino e aprendizagem.

\section{Materiais e Métodos}

A Tecnologia do $Q R$ Code e a série Game of Thrones estão presentes no cotidiano de boa parte dos estudantes. A partir disso, o intuito foi desenvolver uma dinâmica de jogo que despertasse a motivação dos estudantes e que fosse capaz de ajudar a fixar de forma 
VI Congresso Brasileiro de Informática na Educação (CBIE 2017)

Anais do XXIII Workshop de Informática na Escola (WIE 2017)

atrativa e significativa os conteúdos de termometria trabalhados em sala de aula por intermédio dos dispositivos móveis.

A atividade pedagógica teve como sujeitos participantes 18 estudantes do segundo ano do Ensino Médio de uma Escola Estadual de Foz do Iguaçu e teve duração de uma hora/aula. Eles foram orientados previamente pela professora regente a baixarem em seus smarthphones aplicativos leitores $Q R$ Codes, uma vez que a escola fornece wifi e o sistema não suporta muitos aparelhos conectados simultaneamente, deixando-o lento. Cabe salientar que a leitura dos $Q R$ Codes impressos nos cartazes prescinde de internet.

A dinâmica do jogo foi idealizada de forma a utilizar todos os ambientes da escola. As "Casas dos Sete Reinos de Westeros" foram representadas pelos cartazes impressos em folha sulfite A4 afixados em lugares estratégicos com o símbolo (brasão) de cada Casa (Figura 1 (a)) e o seu $Q R$ Code contendo a temperatura e a sua respectiva escala termométrica (Figura 1 (b)). Os $Q R$ Codes foram gerados em formato de texto a partir do site www.waspbarcode.com. Procurou-se fomentar que os estudantes interagissem com os professores e funcionários da escola que estivessem nesses espaços durante a atividade. Os estudantes não tiveram conhecimento prévio da localização dos códigos.

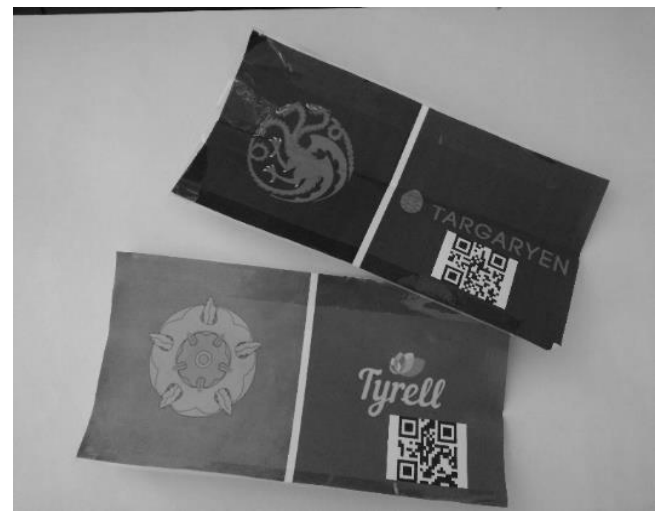

(a)

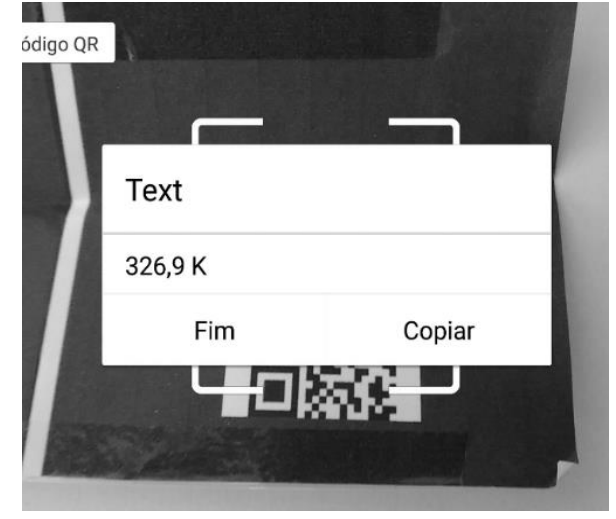

(b)

Figura 1 - Exemplo de cartaz e QR Code produzidos para a atividade

Foram elaborados e impressos cartões em folha sulfite branca tamanho A3 cuja gramatura é maior que a da folha sulfite branca A4. Esses continham em um dos versos o brasão de uma Casa e no outro a sua escala termométrica, como ilustra a Figura 2:

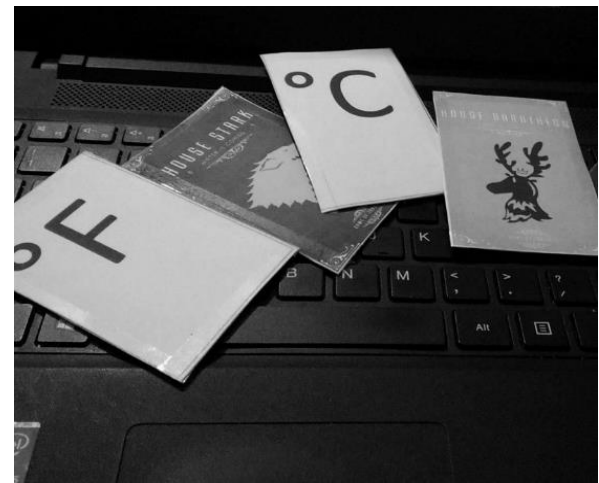

Figura 2 - Exemplo de Cartões utilizadas

Para caçar esses $Q R$ Codes os estudantes tiveram que se orientar pelo mapa adaptado dos Setes Reinos impresso em folha sulfite A4 de cor branca no qual foram 
VI Congresso Brasileiro de Informática na Educação (CBIE 2017)

Anais do XXIII Workshop de Informática na Escola (WIE 2017)

colocados os espaços da escola distribuídos em reinos como se vê na Figura 3:

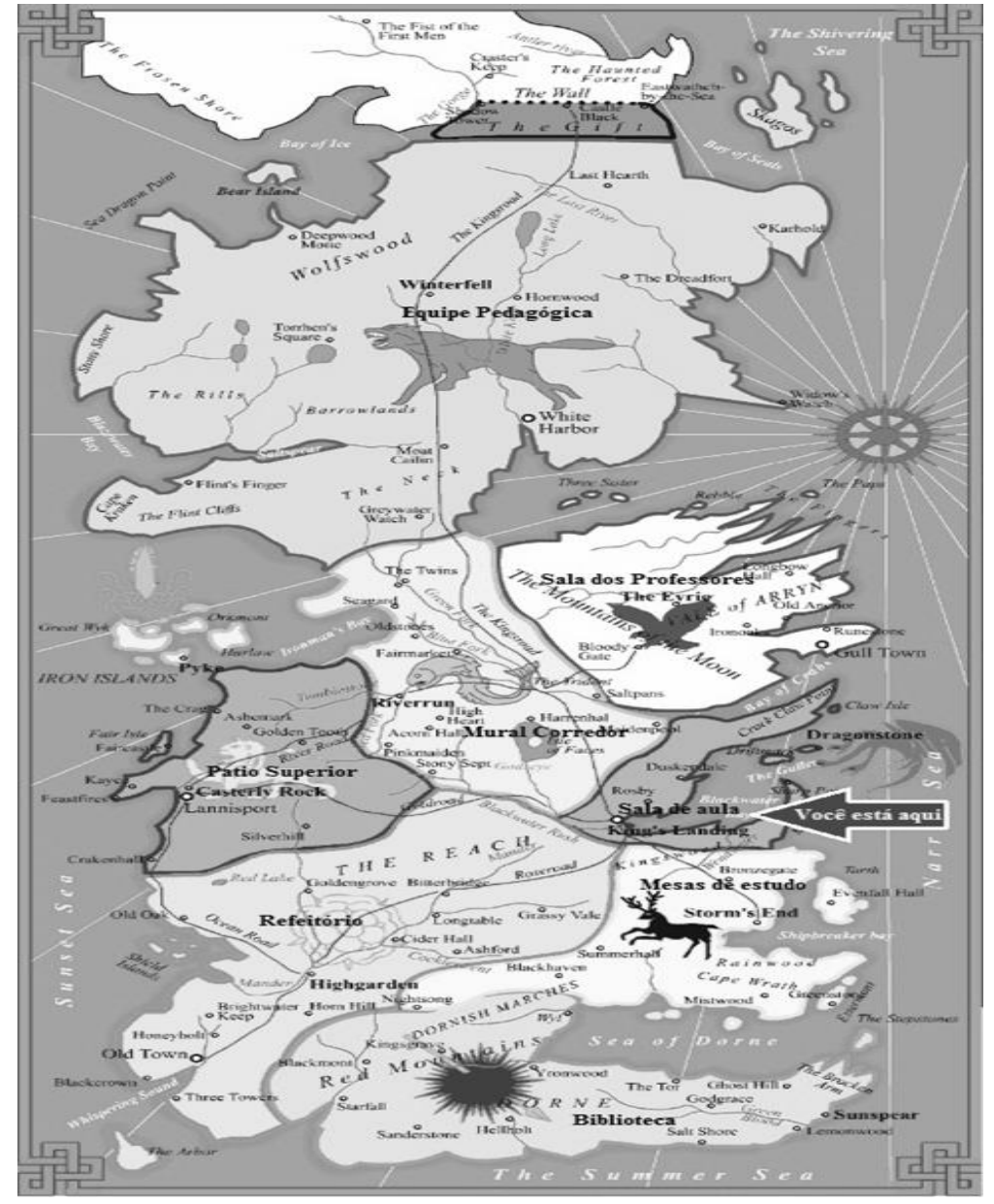

Figura 3 - Mapa adaptado dos Sete Reinos de Westeros

\subsection{Dinâmica de Jogo}

A dinâmica de jogo foi dividida nas etapas: (i) divisão dos grupos e explanação das regras da atividade, (ii) caça aos QR Codes, (iii) disputa dos cálculos, (iv) avaliação da atividade.

A primeira etapa consistiu na divisão dos alunos em 9 duplas (condicionou-se que cada dupla teria pelo menos um smartphone com leitor de $Q R$ Code) e instruções. Cada dupla representou uma Casa dos Setes Reinos: Casa Stark, Casa Greyjoy, Casa Lannister, Casa Tyrell, Casa Baratheon, Casa Martell, Casa Targaryens, Casa Arryn e Casa Tully. Cada dupla tinha um líder, e a dupla a vencedora teria o direito à posse do Trono de Ferro, ou seja, tornar-se-ia em rei ou rainha. Também se explanou sobre a série Game of Thrones, os objetivos da dinâmica e o seu funcionamento. Cada dupla recebeu um mapa (com os setores da escola divididos sobre o mapa dos Sete Reinos de Westeros) e um cartão com sua respectiva Casa e escala. Salientou-se que nos cartazes espalhados pela escola havia $Q R$ Codes com temperaturas e escalas e que ao encontrá-las deveriam capturá-las e copiá-las para a posterior transformação em sala de aula para sua escala termométrica. A cópia poderia ser feita pelo aplicativo leitor de $Q R$ Code ou em papel.

A segunda etapa - "Caça aos $Q R$ Codes - foi a dinâmica em si na qual os estudantes saíram da sala de aula, naquele momento, denominada "Porto Real" - local onde fica o 
Trono de Ferro - para procurarem os cartazes sob orientação mapa. Determinou-se o tempo máximo de 20 minutos para a captura dos $Q R$ Codes com os smartphones e para seguir para a próxima etapa. Dentro desse intervalo, o tempo de captura dependeu da habilidade de cada dupla e das estratégias por elas adotadas.

A terceira etapa - "Disputa dos Cálculos" - consistiu na transformação das escalas termométricas em sala de aula e a "coroação" dos vencedores. Durante a disputa os estudantes estavam autorizados a fazerem alianças que os ajudariam a conquistar o trono. A última etapa consistiu em um bate papo informal no qual os estudantes puderam expor suas impressões sobre a aula gamificada e sobre as atividades realizadas.

Durante toda a realização da dinâmica os professores e pesquisadores observaram e anotaram também suas reflexões e impressões sobre a atividade.

\section{Resultados e Discussões}

Na experiência ora relatada, percebeu-se que a utilização de recursos tecnológicos dos próprios estudantes é uma alternativa que auxilia na superação da falta de infraestrutura tecnológicas das escolas, o que confirma que a perspectiva do BYOD é extremamente eficaz para condução de atividades envolvendo TDIC e, ao mesmo tempo, desperta e motiva os alunos a enxergarem possibilidades de usos pedagógicos de seus smartphones, deixando as aulas mais dinâmicas e interativas.

A atividade exigiu dos estudantes competências e habilidades interdisciplinares como a capacidade de ler mapas, conhecimentos matemáticos e físicos, trabalho em grupo, organização, fluência digital entre outras.

Os estudantes não tiveram dificuldades no manuseio dos smartphones, uma vez que esses aparelhos lhes eram familiares. Por outro lado, notou-se que no momento de converter as temperaturas por intermédio das equações, apresentaram dificuldades com a matemática básica. Dificuldades essas que têm suas raízes no início da vida escolar, conforme relato da professora regente. A matemática é uma área cumulativa de conhecimento, isto é, o aluno precisa aprender adequadamente um conteúdo prévio para compreender o posterior. Dessa maneira, isso significa que uma etapa que não aprendida satisfatoriamente compromete o aprendizado futuro das demais. É importante salientar que para resolução dos cálculos os alunos também utilizaram seus celulares como calculadora, um recurso muito utilizado nas aulas de Física.

No que tange à atuação dos estudantes na atividade gamificada, estes surpreenderam positivamente os pesquisadores, posto que utilizaram estratégias que estão presentes na gamificação e na série Game of Thrones com a aliança entre Casas para a conquista do Trono de Ferro, o que permitiu a colaboração entre os estudantes no momento da captura dos $Q R$ Codes e na transformação das equações termométricas. "Casas" que tinham mais facilidades com as equações auxiliavam as "Casas" aliadas a fazerem a transformação para juntas conquistarem o Trono de Ferro em troca de $Q R$ Codes que lhes faltavam.

No que concerne ao feedback da turma, este foi positivo. Os estudantes relataram que gostaram da atividade e sugeriram que fossem feitas mais atividades como essas. Segue a transcrição do relato de alguns deles: "Eu acharia muito legal se todos os professores tivessem a mesma ideia que você de trazer um jeito contemporâneo pra gente aprender e se divertir. Parabéns, "prof" por ter essa ideia genial”, comentou um dos 
estudantes. Outros relataram que: "a atividade foi bacana uma vez que exigia interação com os colegas"; "foi muito legal por que tinha algumas pessoas da sala que eu não fazia questão de falar e eu tive que deixar meu orgulho de lado e fazer aliança quando necessário". Evidencia-se assim, pelas suas impressões, a carência de atividades de ensino diferenciadas e motivadoras intermediadas pelas tecnologias, não só em Física, mas também nas disciplinas em geral.

A professora regente relatou após a atividade que já havia trabalhado com recursos tecnológicos em sua prática pedagógica, inclusive com $Q R$ Code com outra turma no ano anterior, com resultados positivos no que se refere ao feedback dos estudantes.

Tendo em vista esse panorama, é inequívoco que os dispositivos móveis e as demais TDIC pertencem ao cotidiano da escola e dos estudantes, não há como rechaçálos. São recursos com grandes potencialidades na educação que se apropriados com metodologias e práticas adequadas em sala de aula podem ser sine qua non na motivação, na aprendizagem colaborativa, na fixação de conteúdos etc.

\section{Conclusões}

Tornar o ensino da Física mais interessante e significativo a todos os alunos é um desafio, entretanto, com essa dinâmica de jogo desenvolvida com os estudantes percebeu-se que o uso de tecnologias digitais, de elementos da gamificação, de elementos do cotidiano dos alunos como a série Game of Thones e a experiência com BYOD podem auxiliar neste processo. A partir disso, crê-se que esta atividade alcançou os seus objetivos.

As atividades gamificadas oportunizam aos professores mudarem a concepção de boa parte dos estudantes de que as aulas são desinteressantes e jogos são "legais" por meio da inserção de elementos de jogos a suas aulas. Houve relatos de estudantes que disseram nunca mais esqueceriam as escalas termométricas após a atividade e que apesar de não gostarem de Física devido às operações matemáticas acharam-na mais interessante e divertida. Por conseguinte, esses relatos demonstram que as aulas se tornam mais dinâmicas, além de fomentarem a criatividade e a cooperação entre os educandos.

O uso dos celulares dos estudantes como recursos didáticos é uma alternativa para essa mudança, pois são tecnologias sem custos para a escola. Explorados com metodologias adequadas, os dispositivos móveis podem potencializar a aprendizagem dos estudantes, uma vez que rechaçá-lo no processo de aprendizagem não se mostra mais como uma alternativa na sociedade contemporânea.

Sobre a replicabilidade, acredita-se que a atividade seja reaplicável a outros contextos, uma vez que se utiliza os dispositivos móveis dos próprios estudantes e a infraestrutura existente na escola. Ademais, a tecnologia $Q R$ Code permite a realização de muitas atividades que podem ser atreladas ao ensino e aprendizagem de diversas disciplinas, portanto, espera-se que esta atividade possa inspirar outros educadores.

Como trabalhos futuros, pretende-se a criação de sequências didáticas com $Q R$ Code que possam ser trabalhadas de forma interdisciplinar com outras disciplinas do currículo e que incentivem o uso da aprendizagem móvel no contexto escolar como recurso didático. Sugere-se também como trabalho futuro que a tecnologia $Q R$ Code seja utilizada no armazenamento de informações concernentes ao conteúdo das disciplinas, à vida escolar e seu entorno. Por exemplo, $Q R$ Codes contendo informativos podem ser afixados na escola ou em suas dependências a fim de que a comunidade escolar as 
VI Congresso Brasileiro de Informática na Educação (CBIE 2017)

Anais do XXIII Workshop de Informática na Escola (WIE 2017)

acessem por intermédio dos seus celulares. Na biblioteca, por exemplo, os $Q R$ Codes podem ser implementados nas capas dos livros para que os estudantes ao lê-los obtenham informações sobre o autor, resenhas, resumos sobre o livro de interesse.

\section{Referências}

Analógica instrumentação e controle (2013). "Temperatura: histórico e conceitos". Artigo técnico. Disponível em: http://www.analogica.com.br/arquivos/art002-teperatura-historico-e-conceitos.pdf Acesso em: 13 de março de 2017.

Araújo, D. A. (2015). "O uso de Qr Code no ambiente escolar". Disponível em: http://www.linhadireta.com.br/publico/images/pilares/bbdb2287166d66a4ab2bcf6c7 48ba30a.pdf Acesso em: 28 de março de 2017.

Bastos, D. L. R.; Rapkiewicz, C.; Benvenuti, J. (2016). "Integrando QR Code na educação na EJA: um projeto-piloto voltado para entendimento da língua portuguesa". Anais do XXII Workshop de Informática na Escola, p. 221-230, Uberlândia, MG.

Brandão, D.; Vargas, A. C. (2016). “Avaliação do uso de tecnologias digitais na educação pública”. In: VIVO, Fundação Telefônica;. Experiências Avaliativas de Tecnologias Digitais na Educação. São Paulo: Fundação Telefônica Vivo, 2016. p. 9-16.

Delinski, V. P.; Candido, F. M.; Cerutti, D. M. L.; Celinski, T. M.; Pacheco, M. Z. (2012). "O uso de Qr Code em material didático para o museu virtual da Universidade Estadual de Ponta Grossa". Anais do $10^{\circ}$ Encontro Conversando sobre a Universidade Estadual de Ponta Grossa, Ponta Grossa, PR.

Ferreira, L; Inocencio, A. C.; Júnior, P. P.; Lopes, M. M. (2016). “Gamificação Aplicada ao Ensino de Gerência de Projetos de Software". Anais do XXII Workshop de Informática na Escola, p. 151-160, Uberlândia, MG.

Franco, A. A.; Costa, A. C. M.; Mota, C. V. A.; Gadelha, R. L. L; Graça, R. J. S. (2016). "M-learning: Celulares utilizados como ferramenta didática numa escola pública de Ensino Médio". Anais do XXII Workshop de Informática na Escola, p. 575-582, Uberlândia, MG.

Gamifcación (2013) “¿Qué es la gamificación?”. Elaborada por Gamificación S. L. Disponível em: http://www.gamificacion.com/que-es-la-gamificacion, Espanha. Acesso em: 28 de mar. de 2017.

Lima, R. J.; Silva, J. R. F.; Cruz, J. S.; Santos, M. S. (2015.) “O uso do Qr Code no ensino da Geografia. Anais do V Encontro de Iniciação à Docência da UEPB, p. 1-6, Paraíba.

Marques, N. L R; Araújo, I. S. (2009) "Texto de apoio ao professor de Física: Física Térmica". 20. ed. Porto Alegre: Instituto de Física - UFRGS.

Moraes, C. R.; Varela, S. (2007) "Motivação do aluno durante o processo de ensinoaprendizagem". Revista Eletrônica de Educação. Ano I, n. 1, p. 1-15, Londrina, PR.

Pires, D. P. L; Afonso, J. C.; Chaves, F. A. B. (2006). “A termometria nos séculos XIX e XX”. Revista Brasileira de Ensino de Física, v. 28, n. 1, p. 101-114. São Paulo, SP.

Porvir (2015) "Traga seu próprio dispositivo. Disponível em: http://porvir.org/byodbring-device/ Acesso em 28 de março de 2017. 